\title{
Extremity of the Disordered Phase in the Ising Model on the Bethe Lattice
}

\author{
P. M. Bleher
}

Institute of Applied Mathematics, Academy of Sciences of the USSR, SU-125047 Moscow, USSR

Abstract. We prove that the disordered Gibbs distribution in the ferromagnetic Ising model on the Bethe lattice is extreme for $T \geqq T_{c}^{S G}$, where $T_{c}^{S G}$ is the critical temperature of the spin glass model on the Bethe lattice, and it is not extreme for $T<T_{c}^{S G}$.

\section{Introduction}

The Bethe lattice $\mathscr{T}^{k}$ of degree $k \geqq 1$ is a tree (i.e. a graph without cycles) such that exactly $(k+1)$ edges come out from any of its vertex. The Ising model on the Bethe lattice is defined by the Hamiltonian

$$
H(\sigma)=-\sum_{\langle x, y\rangle} J_{x y} \sigma(x) \sigma(y)
$$

where the sum is taken over all pairs of the nearest neighbors $\langle x, y\rangle$ and the spins $\sigma(x)$ take values \pm 1 .

In the ferromagnetic Ising model

$$
J_{x y} \equiv J>0,
$$

and in the spin glass model the interaction $J_{x y}$ is random and

$$
J_{x y}= \pm J, \quad J>0
$$

with probability $1 / 2$ independently for any pair $\langle x, y\rangle$. Both in the ferromagnetic Ising model and in the spin glass model phase transitions occur, but the values of the corresponding critical temperatures are different. Denote

$$
\theta=\tanh (J / T) .
$$

Then the critical value $\theta$ for the ferromagnetic Ising model is

$$
\theta_{c}^{F}=1 / k
$$


(see [1-9]) and for the spin glass model

$$
\theta_{c}^{S G}=1 / \sqrt{k}
$$

(see [10-12]) where $k$ is the degree of the Bethe lattice.

The question arises whether a manifestation of the spin glass phase transition exists in the ferromagnetic Ising model. The answer to this question turns out to be positive, and as we show in the present work $\theta_{c}^{S G}$ is the stability border of the disordered phase in the ferromagnetic Ising model. The main result of this paper is the following theorem.

Theorem. For $0<\theta \leqq \theta_{c}^{S G}=1 / \sqrt{k}$ and only for these values of $\theta$ the disordered Gibbs distribution is extreme.

Let us remark that for $0<\theta \leqq \theta_{c}^{F}=1 / k$ the Gibbs distribution is unique and so it is extreme. On the other hand in the papers [4] (with a reference to a private communication of Kanae) and [7] it was proved that for $\theta>1 / \sqrt{k}$ the disordered Gibbs distribution is not extreme. Thus our new result is related to the interval $1 / k$ $<\theta \leqq 1 / \sqrt{k}$. This case was discussed in $[7,8]$ as an open problem.

The content of the paper is the following. In Sect. 2, following [1-9], we construct the disordered Gibbs distribution for all values of the temperature. In the main part of this work, in Sect. 3, we describe under which conditions it is extreme. Some results of [11] are essential for this section. In particular, it will be seen that the stability of the disordered Gibbs distributions is closely related to the phase transition in the spin glass model.

Some other bifurcation properties of the disordered Gibbs distribution at the point $\theta=1 / \sqrt{k}$ were studied in $[4,6,7,13]$.

\section{Construction of the Disordered Gibbs Distribution}

Let $V$ and $L$ be respectively the sets of vertices and edges of the graph $\mathscr{T}^{k}$ and $x^{0} \in V$ be an arbitrary vertex. Denote

$$
W_{n}=\left\{x \in V \mid d\left(x, x^{0}\right)=n\right\},
$$

where the distance $d(x, y)$ on $V$ is introduced as the length (the number of edges) of the shortest path connecting $x$ with $y$. It is clear that $W_{n}$ is the "sphere" of radius $n$. Let

$$
V_{n}=\bigcup_{m=l}^{n} W_{m}=\left\{x \in V \mid d\left(x, x^{0}\right) \leqq n\right\}
$$

be the "ball" of radius $n$. Denote $L_{n}=\left\{l=\langle x, y\rangle \in L \mid x, y \in V_{n}\right\}$.

We say that $x<y$ if the path from $x^{0}$ to $y$ goes through $x$. Moreover, $y$ is called a direct successor of $x$ if $y>x$ and $x, y$ are the nearest neighbors. Denote $S(x)$ the set of direct successors of $x$. Observe that any vertex $x \neq x^{0}$ has $k$ direct successors and $x^{0}$ has $(k+1)$ ones.

For $\Lambda \subset V$ denote $\Sigma_{\Lambda}=\{-1,+1\}^{\Lambda}$, the configurational space of the set $\Lambda$. 
Introduce a class of Markov chains on $\mathscr{T}^{k}$. Let $h_{x}$ be a real-valued function of $x \in V$. Consider for each $n$ the probability distribution on $\Sigma_{V_{n}}$, defined by the formula

$$
\mu_{n}\left(\sigma_{n}\right)=Z_{n}^{-1} \exp \left\{(1 / T) \sum_{\langle x, y\rangle \in L_{n}} J_{x y} \sigma(x) \sigma(y)+\sum_{x \in W_{n}} h_{x} \sigma(x)\right\},
$$

where $\sigma_{n}=\left\{\sigma(x), x \in V_{n}\right\} \in \Sigma_{V_{n}}$ and $Z_{n}^{-1}$ is a normalizing factor. We say that the probability distributions $\mu_{n}\left(\sigma_{n}\right)$ are compatible if for all $n \geqq 1$,

$$
\sum_{\sigma^{(n)}} \mu_{n}\left(\sigma_{n-1}, \sigma^{(n)}\right)=\mu_{n-1}\left(\sigma_{n-1}\right),
$$

where $\sigma^{(n)}=\left\{\sigma(x), x \in W_{n}\right\}$. In such a case there exists a Gibbs distribution $\mu$ on $\Sigma_{V}$ such that $\mu\left(\sigma_{n}\right)=\mu_{n}\left(\sigma_{n}\right)$. This Gibbs distribution satisfies the Markov property (see $[3,8])$ and it is called the Markov chain, associated with the interaction $\left\{J_{x y},\langle x, y\rangle \in L\right\}$. The following proposition describes the conditions on the function $h_{x}$ which ensures the compatibility of the probability distributions $\mu_{n}\left(\sigma_{n}\right)$.

Proposition 2.1 (see, e.g. $[3,8]$ ). The probability distributions $\mu_{n}\left(\sigma_{n}\right), n=1,2, \ldots$, in (2.1) are compatible iff for any $x \in V$ the following equation holds:

$$
h_{x}=\sum_{y \in S(x)} \operatorname{artanh}\left(\theta_{x y} \tanh h_{y}\right),
$$

where $\theta_{x y}=\tanh \left(J_{x y} / T\right)$.

It is noteworthy that any extreme Gibbs distribution on $\mathscr{T}^{k}$ corresponding to the interaction $\left\{J_{x y},\langle x, y>L\}\right.$ is a Markov chain (see $\left.[3,4,8]\right)$.

A Markov chain $\mu$ on $\Sigma_{V}$ is called homogeneous if $J_{x y} \equiv J,\langle x, y\rangle \in L$, and $h_{x} \equiv h_{*}$ for any $x=x^{0}$. For $h_{*}(2.3)$ implies the equation

$$
h_{*}=k \operatorname{artanh}\left(\theta \tanh h_{*}\right) \text {, }
$$

$\theta=\tanh (J / T)$. For any $\theta$ this equation has a solution $h_{*}=0$. The homogeneous Markov chain $\mu_{\#}$ corresponding to the solution $h_{*}=0$ is called the disordered Gibbs distribution, or the disordered phase.

Note that in the ferromagnetic case for $\theta>\theta_{c}^{F}=1 / k$ the equation (2.4) has two non-zero solutions $\pm h_{*}, h_{*}>0$, corresponding to two homogeneous extreme Gibbs distributions, namely, + or - phases (see [1, 3, 4, 6-9]). For $\theta>\theta_{c}^{F}$ some non-homogeneous extreme Gibbs distributions of the ferromagnetic Ising model were constructed in $[4,9]$.

\section{Extremity Conditions of the Disordered Phase}

Now we shall prove that the disordered Gibbs distribution of the ferromagnetic Ising model is extreme for $0<\theta \leqq 1 / \sqrt{k}$. We shall verify the following property.

Property $E$. For any $\varepsilon>0, n>0$ and any configuration

$$
\sigma_{n}=\left\{\sigma(x), x \in V_{n}\right\} \in \Sigma_{V_{n}}
$$

there exist $N>n$ and $\Omega_{N} \subset \Sigma_{W_{N}}$ such that

1. $\mu_{\#}\left(\Omega_{N}\right)>1-\varepsilon$;

2. $\left|\mu_{\#}\left(\sigma_{n} \mid \sigma^{(N)}\right)-\mu_{\#}\left(\sigma_{n}\right)\right|<\varepsilon \forall \sigma^{(N)} \in \Omega_{N}$. 
Property E means that for typical boundary conditions $\sigma^{(N)}$ the conditional distributions $\mu_{\#}\left(\sigma_{n} \mid \sigma^{(N)}\right)$ converge to the unconditional ones $\mu_{\#}\left(\sigma_{n}\right)$, as $N \rightarrow \infty$. For the sake of brevity here and later we denote for $A \subset \Sigma_{\Lambda}$,

$$
\mu_{\#}(A)=\mu_{\#}\left(A \times \Sigma_{V \backslash \Lambda}\right),
$$

and for $\sigma_{n} \in \Sigma_{V_{n}}$,

$$
\mu_{\#}\left(\sigma_{n}\right)=\mu_{\#}\left(\left\{\sigma_{n}\right\} \times \Sigma_{V \backslash V_{n}}\right)
$$

Moreover,

$$
\mu_{\#}\left(\sigma_{n} \mid \sigma^{(N)}\right)=\mu_{\#}\left(\sigma_{n}, \sigma^{(N)}\right) / \mu_{\#}\left(\sigma^{(N)}\right),
$$

where

$$
\mu_{\text {\# }}\left(\sigma_{n}, \sigma^{(N)}\right)=\mu_{\text {\#政 }}\left(\left\{\sigma_{n}\right\} \times\left\{\sigma^{(N)}\right\} \times \Sigma_{V \backslash\left(V_{n} \cup W_{N}\right)}\right) .
$$

From Property $\mathrm{E}$ it follows that $\mu_{\#}$ is an extreme Gibbs distribution (see [1]). Let us verify Property E.

Substituting $h_{x}=0, x \in W_{N}$, in (2.1), we have:

$$
\mu_{\#}\left(\sigma_{N}\right)=Z_{N}^{-1} \exp \left\{-(1 / T) H_{N}\left(\sigma_{N}\right)\right\},
$$

where

$$
H_{N}\left(\sigma_{N}\right)=-J \sum_{\langle x, y\rangle \in L_{N}} \sigma(x) \sigma(y),
$$

$\sigma_{N}=\left\{\sigma(x), x \in V_{N}\right\}$. This formula can be interpreted in the following way: If

$$
h_{x}^{(N)}=\frac{J}{T} \sum_{y \in S(x)} \sigma(y), \quad x \in W_{N-1},
$$

then

$$
\begin{aligned}
H_{N}\left(\sigma_{N}\right) & =H_{N-1}\left(\sigma_{N-1}\right)-J \sum_{x \in W_{N-1}} \sigma(x) \sum_{y \in S(x)} \sigma(y) \\
& =H_{N-1}\left(\sigma_{N-1}\right)-T \sum_{x \in W_{N-1}} h_{x}^{(N)} \sigma(x),
\end{aligned}
$$

so

$$
\mu_{\#}\left(\sigma_{N}\right)=Z_{N}^{-1} \exp \left\{-(1 / T) H_{N-1}\left(\sigma_{N-1}\right)+\sum_{x \in W_{N-1}} h_{x}^{(N)} \sigma(x)\right\} .
$$

This implies that the joint distribution of the random variables

$$
\left\{\sigma(x), x \in V_{N-1}\right\}=\sigma_{N-1}
$$

and $\left\{h_{x}^{(N)}, x \in W_{N-1}\right\}=h^{(N, N-1)}$ with respect to $\mu_{\text {\# }}$ has the form

$$
\begin{aligned}
\mu_{\#}\left(\sigma_{N-1}, h^{(N, N-1)}\right) & =\sum_{\bar{T}_{y \in S(x)} \sum_{\sigma(y)=h_{x}^{(N)}, x \in W_{N-1}}} \mu_{\#}\left(\sigma_{N}\right) \\
& =\tilde{Z}_{N}^{-1} \exp \left\{-(1 / T) H_{N-1}\left(\sigma_{N-1}\right)\right. \\
& \left.+\sum_{x \in W_{N-1}} h_{x}^{(N)} \sigma(x)\right\}_{x \in W_{N-1}} v\left(h_{x}^{(N)}\right),
\end{aligned}
$$


where $v\left(h_{x}^{(N)}\right)$ is the distribution of the random variable (3.1) under the condition that $\sigma(y)$ are independent, $\sigma(y)= \pm 1$ with probability $1 / 2$. It is easy to see that $v$ is the binomial distribution.

Formula (3.3) resembles (2.1) but now the variables $h_{x}^{(N)}$ are random. Using the recurrent equations

$$
h_{x}^{(N)}=\sum_{y \in S(x)} \operatorname{artanh}\left(\theta \tanh h_{y}^{(N)}\right),
$$

where $\theta=\tanh (J / T)$ define the set of random variables

$$
\left\{h_{x}^{(N)}=h_{x}^{(N)}\left(\sigma^{(N)}\right), x \in V_{N-1}\right\} .
$$

Since the random variables $h_{x}^{(N)}$ satisfy the compatibility conditions (3.4), Proposition 2.1 implies that the joint distribution of the random variables

$$
\left\{\sigma(x), x \in V_{n}\right\}=\sigma_{n} \text { and }\left\{h_{x}^{(N)}, x \in W_{n}\right\}=h^{(N, n)}
$$

with respect to $\mu_{\#}$ has the form

$$
\begin{gathered}
\mu_{\text {\# }}\left(\sigma_{n}, h^{(N, n)}\right)=Z_{N, n}^{-1} \exp \left[-(1 / T) H_{n}\left(\sigma_{n}\right)\right. \\
\left.+\sum_{x \in W_{n}} h_{x}^{(N)} \sigma(x)\right\}_{x \in W_{n}} v_{N-n}\left(h_{x}^{(N)}\right),
\end{gathered}
$$

where the probability distribution $v_{N-n}\left(h_{x}^{(N)}\right)$ is defined in the following way. Consider the set of independent random variables $\left\{\sigma(x), x \in W_{N}\right\}$ taking values \pm 1 with probability $1 / 2$, and the corresponding probability space $\left(\Sigma_{W_{N}}, B, \mu_{0}\right)$, where $\mu_{0}$ is the Bernoulli measure with $p=q=1 / 2$. Consider on this probability space the random variables $h_{x}^{(N)}$ which are defined recurrently by Eqs. (3.2), (3.4). Then for any fixed $n<N$ the random variables $\left\{h_{x}^{(N)}, x \in W_{n}\right\}$ are independent, identically distributed and symmetric. By $v_{N-n}\left(h_{x}^{(N)}\right)$ we denote the distribution of $h_{x}^{(N)}$ for $x \in W_{n}$.

The central point of our considerations is the following lemma. Let $D h_{x}^{(N)}$ denote the variance of the random variable $h_{x}^{(N)}=h_{x}^{(N)}\left(\sigma^{(N)}\right)$ with respect to the Bernoulli measure $\mu_{0}$.

Lemma 3.1 (see [11]). If $0<\theta \leqq \theta_{c}^{S G}=1 / \sqrt{k}$, then

$$
\lim _{N \rightarrow n \rightarrow \infty} D h_{x}^{(N)}=0, \quad x \in W_{n} .
$$

If $\theta_{c}^{S G}<\theta<1$, then there exists some $\varepsilon>0$ such that

for any $N>n \geqq 0$.

$$
D h_{x}^{(N)}>\varepsilon, \quad x \in W_{n},
$$

We remark that this lemma plays an essential rôle in the proof of the existence of the spin-glass phase transition on the Bethe lattice (see [11]). It is proved in [11] only for $k=2$, but the generalization to the case $k>2$ is straightforward. For the sake of completeness we present a simplified version of the proof of Lemma 3.1 in the case most interesting for us, when $\theta \leqq 1 / \sqrt{k}$.

From the independence of $h_{y}^{(N)}$ in (3.4) it follows that

$$
D h_{x}^{(N)}=k D \operatorname{artanh}\left(\theta \tanh h_{y}^{(N)}\right),
$$


$x \in W_{m-1}, y \in W_{m}, m \leqq N-1$. It is easy to verify that for any $t \in \mathbb{R}^{1}$,

$$
|\operatorname{artanh}(\theta \tanh t)| \leqq \theta|t|
$$

so we get

$$
D h_{x}^{(N)} \leqq k \theta^{2} D h_{y}^{(N)}
$$

Iterating this inequality we have that for $x \in W_{n}, y \in W_{N-1}, n \leqq N-1$,

$$
D h_{x}^{(N)} \leqq\left(k \theta^{2}\right)^{N-1-n} D h_{y}^{(N)},
$$

which implies (3.6) for $\theta<1 / \sqrt{k}$.

For $\theta=1 / \sqrt{k}$ we have to refine the above arguments since (3.7) ensures in this case only that

$$
D h_{x}^{(N)} \leqq D h_{y}^{(N)},
$$

$x \in W_{m-1}, y \in W_{m}$, i.e. that with the decrease of $m$ the value $D h_{x}^{(N)}$ does not increase. Assume that

$$
\lim _{N-n \rightarrow \infty} D h_{x}^{(N)}=\gamma>0, \quad x \in W_{n}
$$

Then for all $N>n \geqq 0$,

$$
D h_{x}^{(N)} \geqq \gamma>0, \quad x \in W_{n} .
$$

Let $\zeta$ be a real symmetric random variable and let $F(t)$ denote its distribution function. For $\varepsilon>0$ introduce

$$
D_{\varepsilon} \zeta=\int_{|t|>\varepsilon} t^{2} d F(t),
$$

i.e. the "cut-off" variance of $\zeta$.

Observe that for $|t|>\varepsilon$,

$$
\left|\operatorname{artanh}\left(\frac{1}{\sqrt{k}} \tanh t\right)\right|<\frac{1}{\sqrt{k}}(1-\delta)|t|
$$

with some $\delta=\delta(\varepsilon)>0$. Thus for $x \in W_{m-1}, y \in W_{m}$,

$$
\begin{aligned}
D h_{x}^{(N)} & =k D \operatorname{artanh}\left(\frac{1}{\sqrt{k}} \tanh h_{y}^{(N)}\right) \\
& \leqq k\left\{\sum_{|t| \leqq \varepsilon} \frac{t^{2}}{k} v_{N-m}(t)+\sum_{|t|>\varepsilon} \frac{t^{2}}{k}(1-\delta)^{2} v_{N-m}(t)\right\} \\
& =\left(D h_{y}^{(N)}-D_{\varepsilon} h_{y}^{(N)}\right)+(1-\delta)^{2} D_{\varepsilon} h_{y}^{(N)} \\
& \leqq\left(D h_{y}^{(N)}-D_{\varepsilon} h_{y}^{(N)}\right)+(1-\delta) D_{\varepsilon} h_{y}^{(N)}=D h_{y}^{(N)}-\delta D_{\varepsilon} h_{y}^{(N)} \\
& =D h_{y}^{(N)}\left(1-\delta D_{\varepsilon} h_{y}^{(N)} / D h_{y}^{(N)}\right) .
\end{aligned}
$$

Moreover, since for any $t \in \mathbb{R}^{1},|t|<1$,

$$
|\operatorname{artanh}(\theta t)| \leqq \operatorname{artanh} \theta=J / T,
$$


by (3.2), (3.4)

$$
\left|h_{y}^{(N)}\right| \leqq k J / T
$$

for any $y \in V_{N-1}$. Hence

$$
D h_{y}^{(N)} \leqq(k J / T)^{2},
$$

so by (3.10)

$$
D h_{x}^{(N)} \leqq D h_{y}^{(N)}\left(1-\delta_{0} D_{\varepsilon} h_{y}^{(N)}\right),
$$

where $\delta_{0}=\delta /(k J / T)^{2}$. Iterating this inequality we get that

$$
D h_{x}^{(N)} \leqq D h_{y}^{(N)} \prod_{z: x<z \leqq y}\left(1-\delta_{0} D_{\varepsilon} h_{z}^{(N)}\right) .
$$

Comparing the last inequality with (3.9) we get that

$$
\lim _{N \rightarrow n \rightarrow \infty} D_{\varepsilon} h_{x}^{(N)}=0, \quad x \in W_{n} .
$$

This relations hold for any $\varepsilon>0$ and

$$
D h_{x}^{(N)} \leqq \varepsilon^{2}+D_{\varepsilon} h_{x}^{(N)} .
$$

Thus $\lim _{N-n \rightarrow \infty} D h_{x}^{(N)}=0$, which proves (3.6) for $\theta=1 / \sqrt{k}$.

The proof of (3.7) for $\theta>1 / \sqrt{k}$ is in [11].

Let us note once more that the different behavior of $D h_{x}^{(N)}, x \in W_{n}$, as $N-n \rightarrow \infty$, for $\theta \leqq 1 / \sqrt{k}$ and for $\theta>1 / \sqrt{k}$ is a manifestation of the phase transition occurring in the spin glass model on the Bethe lattice (see details in [10-12]). the set

Let $0<\theta \leqq 1 / \sqrt{k}$ and $n>0, \delta>0$ be fixed. To verify Property E define for $N>n$

$$
\Omega_{N, \delta}=\left\{\sigma^{(N)}=\left\{\sigma(x), x \in W_{N}\right\}|| h_{x}^{(N)}\left(\sigma^{(N)} \mid \leqq \delta, x \in W_{n}\right\} .\right.
$$

Let us prove that

$$
\lim _{N \rightarrow \infty} \mu_{\#}\left(\Omega_{N, \delta}\right)=1
$$

By (3.5)

$$
\mu_{\#}\left(\Omega_{N, \delta}\right)=\frac{\sum_{\left\{h_{x}^{(N)} \mid \leq \delta, x \in W_{n}\right\}} Q_{n}\left(\left\{h_{x}^{(N)}, x \in W_{n}\right\}\right)}{\sum_{\left\{h^{(N)}, x \in W_{n}\right\}} Q_{n}\left(\left\{h_{x}^{(N)}, x \in W_{n}\right\}\right)},
$$

where

$$
Q_{n}\left(\left\{h_{x}^{(N)}, x \in W_{n}\right\}\right)=\sum_{\sigma_{n}} \exp \left\{-(1 / T) H_{n}\left(\sigma_{n}\right)+\sum_{x \in W_{n}} h_{x}^{(N)} \sigma(x)\right\} \prod_{x \in W_{n}} v_{N-n}\left(h_{x}^{(N)}\right) .
$$

By Lemma 3.1

$$
\lim _{N \rightarrow \infty} h_{x}^{(N)}=0, \quad x \in W_{n},
$$

when $n$ is fixed and $\theta \leqq 1 / \sqrt{k}$, so (3.12) follows from (3.13). 
Let us estimate now $\left|\mu_{\#}\left(\sigma_{n} \mid \sigma^{(N)}\right)-\mu_{\#}\left(\sigma_{n}\right)\right|$. By (3.5) we have that

$$
\mu_{\#}\left(\sigma_{n} \mid \sigma^{(N)}\right)=\frac{\exp \left\{-(1 / T) H_{n}\left(\sigma_{n}\right)+\sum_{x \in W_{n}} h_{x}^{(N)}(x)\right\}}{\sum_{\sigma_{n}} \exp \left\{-(1 / T) H_{n}\left(\sigma_{n}\right)+\sum_{x \in W_{n}} h_{x}^{(N)} \sigma(x)\right\}},
$$

where $h_{x}^{(N)}=h_{x}^{(N)}\left(\sigma^{(N)}\right), x \in W_{n}$, are expressed via $\sigma^{(N)}$ by formulas (3.2), (3.4). Moreover according to (3.1),

$$
\mu_{\#}\left(\sigma_{n}\right)=\frac{\exp \left\{-(1 / T) H_{n}\left(\sigma_{n}\right)\right\}}{\sum_{\sigma_{n}} \exp \left\{-(1 / T) H_{n}\left(\sigma_{n}\right)\right\}} .
$$

As $n$ is fixed, it follows obviously from (3.14), (3.15) that for any $\varepsilon>0$ there exists $\delta>0$ such that

$$
\mid \mu_{\#}\left(\sigma_{n} \mid \sigma^{(N)}\right)-\mu_{\#}\left(\sigma_{n}\right)<\varepsilon,
$$

if $\left|h_{x}^{(N)}\right|<\delta$ for all $x \in W_{n}$, i.e. for $\sigma^{(N)} \in \Omega_{N, \delta}$.

Thus for a given $\varepsilon>0$ we can choose at first such $\delta>0$ that (3.16) is fulfilled for $\sigma^{(N)} \in \Omega_{N, \delta}$, and next by (3.12) such $N$ that

$$
\mu_{\#}\left(\Omega_{N, \delta}\right)>1-\varepsilon .
$$

Hence we have proved that for $\mu_{\#}$ Property $\mathrm{E}$ is valid, so $\mu_{\#}$ is an extreme Gibbs distribution for $\theta \leqq 1 / \sqrt{k}$.

Let $\theta>1 / \sqrt{k}$. Then (3.7) holds for some $\varepsilon>0$. Taking into account the estimate (3.11) we get that there exists some $\delta>0$ such that

$$
\operatorname{Prob}\left\{\left|h_{x}^{(N)}\right|>\delta\right\}>\delta, \quad x \in W_{n},
$$

for all $N>n \geqq 0$. As the random variable $h_{x}^{(N)}$ is symmetric, this implies that

$$
\operatorname{Prob}\left\{h_{x}^{(N)}>\delta\right\}>\delta / 2, \quad x \in W_{n} .
$$

Introduce the set

$$
\Lambda_{N, \delta}=\left\{\sigma^{(N)} \mid h_{x^{0}}^{(N)}\left(\sigma^{(N)}\right)>\delta\right\}
$$

Then it is easy to see from formulas (3.13)-(3.15) that there exists some $\gamma>0$ such that for any $N>0$,

$$
\mu_{\#}\left(\Lambda_{N, \delta}\right) \geqq \gamma
$$

and

$$
\left|\mu_{\#}\left(\sigma\left(x^{0}\right) \mid \sigma^{(N)}\right)-\mu_{\#}\left(\sigma\left(x^{0}\right)\right)\right| \geqq \gamma, \quad \sigma^{(N)} \in \Lambda_{N, \delta} .
$$

This means that Property E does not hold which implies that the distribution $\mu_{\#}$ is not extreme (see [1]). The theorem is proved. 


\section{References}

1. Preston, C.J.: Gibbs states on countable sets. Cambridge Tracts in Mathematics, vol. 68. London: Cambridge University Press 1974

2. Katsura, S., Takizawa, M.: Bethe lattice and the Bethe approximation. Progr. Theor. Phys. 51, 82-98 (1974)

3. Spitzer, F.: Markov random fields on an infinite tree. Ann. Prob. 3, 387-398 (1975)

4. Higuchi, Y.: Remarks on the limiting Gibbs states on a $(d+1)$-tree. Publ. RIMS Kyoto University 13, 335-348 (1977)

5. Baxter, R.J.: Exactly solved models in statistical mechanics. London, New York: Academic Press 1982

6. Falk, H.: Ising spin system on a Cayley tree: correlation decomposition and phase transition. Phys. Rev. B 12, 5184-5189 (1975)

7. Moore, T., Snell, J.L.: A branching process showing a phase transition. J. Appl. Prob. 16, 252-260 (1979)

8. Georgii, H.-O.: Gibbs measures and phase transitions (De Gruyter Studies in Math., vol. 9). Berlin, New York: De Gruyter 1988

9. Bleher, P.M., Ganihodgaev, N.N.: On pure phases of the Ising model on the Bethe lattice (to appear in Theor. veroyatn. primen.)

10. Thouless, D.J.: Spin-glass on a Bethe lattice. Phys. Rev. Lett. 56, 1082-1085 (1986)

11. Chayes, J.T., Chayes, L., Sethna, J.P., Thouless, D.J.: A mean field spin glass with short-range interactions. Commun. Math. Phys. 106, 41-89 (1986)

12. Carlson, J.M., Chayes, J.T., Chayes, L., Sethna, J.P., Thouless, D.J.: Critical behavior of the Bethe lattice spin glass. Europhys. Lett. 5, 355-360 (1989)

13. Matsuda, H.: Infinite susceptibility without spontaneous magnetization. Progr. Theor. Phys. 51, 1053-1063 (1974)

Communicated by Ya. G. Sinai

Received April 1, 1989 
